\title{
A Disciplina História da Matemática do CuRso de Matemática da UNIVERSIDADE ESTADUAL DE MARINGÁ, ANOS 1972-1982
}

\author{
Suélen Rita Andrade Machado \\ Faculdade de Engenharia e Inovação Técnico Profissional - FEITEP - Brasil \\ Lucieli M. Trivizoli \\ Universidade Estadual de Maringá - UEM - Brasil
}

(aceito para publicação em maio de 2020)

\begin{abstract}
Resumo
A História da Matemática vem se consolidando como uma disciplina autônoma desde o século XX. Entretanto, o que se sabe sobre sua trajetória histórica no Brasil ainda é limitada, pois são poucas as pesquisas históricas que retratam tal objeto investigativo. Tendo em vista esses indícios, nesse artigo apresentamos uma caracterização histórica da presença da disciplina História da Matemática no primeiro currículo do curso de Matemática da Universidade Estadual de Maringá - UEM, nos anos 1972 a 1982. Para obtenção dos dados, nos valemos de fontes primárias do Inventário Arquivístico desse curso e, então, produzimos uma narrativa descritiva acerca da presença histórica dessa disciplina, baseada na análise documental. Os resultados apontam uma inclusão curricular breve, influenciada por outros cursos de Matemática do período e uma abordagem do conteúdo sem conexões com aspectos didático-pedagógicos formativos.
\end{abstract}

Palavras-chave: Currículo, Disciplina, História da Educação Matemática.

\section{[The Discipline History OF MATHEMATICS OF THE MATHEMATICS COURSE OF THE MARINGÁ STATE UNIVERSITY, 1972-1982]}

\begin{abstract}
The History of Mathematics has been consolidated as an autonomous academic discipline since the twentieth century. What is known about its historical trajectory in Brazil still is limited, because there are only few historical researches that portray such an investigative
\end{abstract}


object. Considering these indications, in this article we present a historical characterization of the presence of the History of Mathematics discipline in the first curriculum of the Mathematics course of the State University of Maringá - UEM, from 1972 to 1982. To obtain the data, we use primary sources from the Archival Inventory of this course and then, we produced a descriptive narrative about the historical presence of this discipline, based on documental analysis. The results show a brief curricular inclusion, influenced by other Mathematics courses of the period and a content approach without connections with formative didactic-pedagogical aspects.

Keywords: Curriculum, Academic discipline, History of Mathematical Education.

\section{Introdução}

Não se sabe ao certo se a História da Matemática começou a ser ensinada ainda no século XVIII, como disciplina, o que se sabe, são elementos que indicam a prescrição de aspectos históricos da Matemática para o tratamento do elenco de disciplinas de um dos currículos mais antigos de Matemática, proposto pela Faculdade de Matemática da Universidade de Coimbra, em 1772. A recomendação era introduzir aspectos da História da Matemática, à medida que os alunos avançassem no curso, porém limitações ocorreram em virtude da falta de profundidade de conhecimentos matemáticos específicos tanto de alunos quanto de professores (SILVA, 2001).

Apesar dessa iniciativa, apenas no III Congresso Internacional de Matemática, na Alemanha, em 1904, surgiu "[...] uma recomendação forte para a introdução da História da Matemática como disciplina indispensável à formação de professores” (SILVA, 2001, p. 132), e alguns matemáticos como Félix Klein, passaram a se dedicar e a recomendar o ensino da História da Matemática internacionalmente. Conforme Jones (1969), outras recomendações de inclusão dessa disciplina foram prescritas por relatórios de comitês de muitos países. Isso corroborou para que no século $\mathrm{XX}$, a disciplina se tornasse autônoma nos meios acadêmicos (NOBRE, 2002). Ainda, de acordo com Wussing (1991) ao comentar sobre o desenvolvimento da História das Ciências a partir de 1960, destaca que:

"o rápido desenvolvimento das ciências, incluindo a matemática, foi acompanhado nos últimos anos por um aumento da história da matemática e da história das ciências. Ambas deixaram o papel de meros hobbies ou preferências anos atrás e se tornaram disciplinas científicas autônomas, com suas próprias perguntas, métodos e resultados" (WUSSING, 1991, p. 64, tradução nossa).

Em relação ao Brasil, pouco se sabe sobre a história da inclusão dessa disciplina nos currículos dos cursos de graduação em Matemática (SILVA, 2001). Em um breve levantamento realizado simultaneamente no Banco de Teses e Dissertações da CAPES e na 
Biblioteca Digital Brasileira de Teses e Dissertações - BDTD, a partir da palavra-chave "disciplina História da Matemática", constatamos dentre os doze trabalhos encontrados, ${ }^{1}$ apenas a dissertação de Stamato (2003) que investigou historicamente a implantação da disciplina História da Matemática nas Licenciaturas em Matemática na UNESP, campi de Rio Claro, Rio Preto e Presidente Prudente e a dissertação de Fragoso (2011) que analisou historicamente a inserção da disciplina História da Matemática no currículo do curso de Licenciatura em Matemática da Universidade Federal de Juiz de Fora - UFJF. O que indica, que ainda há poucas pesquisas que trazem a história dessa disciplina nos cursos de Matemática no país.

Ciente desse fato e considerando o levantamento realizado por Stamato (2003) acerca das instituições brasileiras que no início do século XXI não prescreviam no currículo do curso de Matemática a disciplina História da Matemática, encontramos dentre aquelas instituições, o curso de Matemática da Universidade Estadual de Maringá. Conforme Machado (2019), no currículo atual desse curso, a disciplina ainda não faz parte do ementário com o nome explícito de História da Matemática, mas permeia uma das disciplinas de Teoria e Prática Pedagógica, fazendo parte de seu programa de ensino. Porém, a pesquisadora adverte que a disciplina de História da Matemática constava no currículo daquele curso nos anos de 1972 a 1982, quando foi excluída do ementário curricular.

Tendo em vista esse episódio histórico, nos questionamos sobre: - Quando a disciplina História da Matemática foi incluída no currículo do Curso de Matemática da Universidade Estadual de Maringá? Quando foi ministrada? Como era o ementário? Quem foram os professores? Houve alunos matriculados? Que tipo de atividade era realizada? Quanto tempo foi prescrita? Quando e por que foi excluída do ementário?

De modo a responder tais questionamos, no presente artigo buscamos caracterizar historicamente a presença da disciplina História da Matemática no primeiro currículo do curso de Matemática da Universidade Estadual de Maringá - UEM, nos anos 1972 a 1982, baseadas em fontes primárias do inventário arquivístico do curso de Matemática e em fontes secundárias correlatas a investigações relacionadas ao histórico e ao currículo do curso de Matemática da Universidade Estadual de Maringá.

Por se constituir como pesquisa histórica, sua natureza é qualitativa pois toma como foco a compreensão e a interpretação de dados e discursos documentais (D’AMBROSIO, 2004), precisamente, de fontes primárias do Departamento de Matemática da Universidade Estadual de Maringá, que não receberam nenhum tratamento analítico e de fontes secundárias correlatas ao tema que tratamos. Para análise das fontes documentais e construção da caracterização histórica, construíremos uma narrativa, pois segundo Cury (2010) elas corroboram para preservação de memórias históricas.

Outrossim, também utilizaremos os procedimentos apresentados por Trivizoli (2016) para o desenvolvimento e a compreensão dos documentos históricos. Haja vista,

\footnotetext{
${ }^{1}$ Encontramos a partir da palavra-chave: Cinco trabalhos no Banco da CAPES e Sete trabalhos na BDTD. A partir dos procedimentos metodológicos adotados por Cervo, Bervian e Silva (2007) de pré-leitura, leitura seletiva e interpretativa, constatamos apenas dois trabalhos que se relacionavam a presença histórica da disciplina em cursos de graduação brasileiros.
} 
segundo ela, que um episódio histórico é oriundo de diferentes processos, que podem ser analisados sob diversas óticas. Isso consiste na natureza da complexidade histórica, como uma variação das influências do contexto, das próprias ideias e interesses dos historiadores. Assim, para a caracterização da disciplina, para compreensão da história documentada utilizaremos o processo dinâmico denominado pela pesquisadora de zooming in, que foca em detalhes específicos, e zooming out, que olha "[...] para uma perspectiva mais ampla, analisando influências culturais, sociais, políticas e científicas contemporâneas e ou relacionadas ao evento em estudo" (TRIVIZOLI, 2016, p. 191). Entendemos, que a conexão entre tais procedimentos, corrobora para construção de uma possível história para essa disciplina.

Nesse sentido, para as próximas seções apresentaremos alguns pressupostos para inserção da disciplina História da Matemática no currículo da UEM, a trajetória dessa disciplina nessa instituição e algumas considerações acerca desse objeto investigativo.

\section{Pressupostos para inserção da disciplina História da Matemática no currículo da UEM}

A escassez de pesquisas históricas relativas ao histórico da disciplina de História da Matemática no currículo dos cursos brasileiros dificulta a escrita em detalhes acerca da temática. Assim, essas lacunas nos conduzem a emprestar o termo zooming out de Trivizoli (2016) para considerar perspectivas mais amplas, relacionadas a variantes culturais, sociais, políticos relacionados ao objeto histórico escolhido.

Os primeiros indicativos acerca da presença da disciplina História da Matemática em um currículo do Ensino Superior Brasileiro datam da década de 1930, precisamente junto a criação do curso de Matemática da Universidade de São Paulo - USP, em 1934. Anterior a isso, uma eclosão para inserção de aspectos históricos da matemática já acontecia, amparada por meio da Reforma do Ensino Secundário Brasileiro - Decreto $\mathrm{n}^{\mathrm{o}}$ 19.890 de 1931, o que pode ter influenciado a inserção da disciplina História da Matemática no currículo de um dos primeiros cursos de Matemática do Brasil, que se diferenciava dos demais cursos por preocupar-se com a formação de matemáticos e professores de matemática especializados (SILVA, 2001; TRIVIZOLI, 2011; CAVALARI, 2012). Entretanto, Silva (2001) destaca que são desconhecidas informações sobre a oferta dessa disciplina no início do curso, apesar da prescrição.

Do período destacado até a década de 1970, pouco se sabe sobre a inserção dessa disciplina nos currículos brasileiros do Ensino Superior, o que se sabe são indícios históricos dispersos pelo país, em virtude do Movimento da Matemática Moderna, que apreciava a abordagem tecnicista do ensino e deixava de lado a abordagem histórica dos conteúdos. Nesse panorama, no ano de 1962, mais de sessenta matemáticos canadenses e norte-americanos assinaram um manifesto que advertia prejuízos pedagógicos ao se desconsiderar aspectos históricos da matemática e por apenas considerar uma abordagem formal da matemática. Tais fatos, contribuíram para uma abrangência explícita da História da Matemática nos livros didáticos, conforme Stamato (2003), e acreditamos que tenha influenciado a composição curricular dos cursos superiores de Matemática no país. 
No Estado do Paraná, por exemplo, verifica-se a inclusão da disciplina História da Matemática como obrigatória no curso de Matemática da Faculdade de Filosofia, Ciências e Letras da Universidade do Paraná na década de 1960 (STAMATO, 2003). Já no Estado de São Paulo, em virtude de uma reforma curricular no Instituto de Matemática (IME), em 1968

“(...) a disciplina História da Matemática passou a fazer parte do elenco de disciplinas obrigatórias do currículo da licenciatura de Matemática da USP. Todavia, como relata Elza Gomide, a oferta dessa disciplina passou por várias dificuldades. A primeira delas foi a ausência de bibliografia em língua portuguesa e a segunda e fundamental, a falta de professores preparados para ministrá-la. Para sanar a primeira dificuldade, Elza Gomide traduziu o livro History of Mathematics de Carl Boyer, que foi editado em 1972. Essa obra passou a ser utilizada como livro-texto na disciplina de História da Matemática, ofertada na USP" (SILVA, 2001, pp. 144-145, grifo nosso).

Entendemos que a inclusão curricular dessa disciplina nesses currículos pode ter suscitado a mesma ideia em outros cursos de Matemática do Brasil, como por exemplo, no curso de Matemática da Universidade Estadual de Maringá.

\section{A trajetória histórica da disciplina na UEM}

No ano de 1971 foi criado o curso de Matemática da Universidade Estadual de Maringá a fim de suprir a carência de professores na região Noroeste do Paraná, constituir corpo docente de estabelecimentos de ensino oficiais e particulares de Maringá e "abrir portas" aos alunos para os cursos de Engenharia recém implantados na instituição (ARAUJO NETO, 2016; D’ANTONIO, 2013).

Naquele mesmo ano foi prescrito o primeiro currículo do curso de Matemática para vigência nos anos $1971-1978,{ }^{2}$ determinado politicamente pelo currículo mínimo para cursos de Matemática, cujos pareceres do Conselho Federal de Educação nºs. 292 e 295 de 1962 regulamentavam sua homologação (MACHADO; TRIVIZOLI, 2018). Aquele currículo foi influenciado, também, por diversas grades curriculares de cursos de Matemática de outras instituições no país, segundo relato do professor Amaury Meller para Araujo Neto (2016).

Entretanto, dentre as disciplinas do currículo mínimo e as escolhidas pelo Departamento de Matemática da UEM, a disciplina História da Matemática ainda não havia sido incluída no rol de disciplinas. De acordo com Machado (2019), a inclusão dessa disciplina ocorreu a partir do $2^{\circ}$ Semestre de 1973 , em virtude de uma alteração curricular

${ }^{2}$ De outra parte, destaca-se modificações curriculares nesse currículo, dentre elas a que incluiu a disciplina História da Matemática no rol de disciplinas. Uma análise das modificações curriculares desse curso, entre os anos 1971 a 1996, pode ser verificada em Machado (2019).

RBHM, Vol. 20, n 39, pp. 63-75, 2020 
do curso para se adequar as políticas educacionais que eclodiam naquele momento, às necessidades do curso e da própria instituição.

Consideramos que a inclusão da disciplina História da Matemática nesse currículo da UEM fora, de certa forma, influência da reforma curricular implementada pelo Instituto de Matemática da Universidade Estadual de São Paulo - USP, em 1968, que incluía no currículo de Licenciatura em Matemática a obrigatoriedade da disciplina História da Matemática. Indícios dessa influência podem ser encontrados em Stamato (2013) e em Araujo Neto (2016), que dialogam sobre certa "[...] convergência nas concepções e criação dos cursos de Matemática do Estado de São Paulo e da UEM com o curso da USP" (ARAUJO NETO, 2016, p. 63). Além disso, também podemos citar a presença e obrigatoriedade da disciplina de História da Matemática, desde a década de 1960, na Faculdade de Filosofia, Ciências e Letras da Universidade do Paraná, atual Universidade Federal do Paraná - UFPR, que poderia ser uma influência para a prescrição curricular na UEM, uma vez que tal instituição se situava também no Estado do Paraná (STAMATO, 2013).

Assim, sobre a inclusão da Disciplina de História da Matemática para o primeiro currículo do curso da UEM, consideramos que sua inserção foi fruto da influência de outras universidades, uma vez, como já salientamos, a primeira grade curricular do curso foi resultante de outros currículos de Cursos de Matemática de instituições superiores do Brasil. Entretanto, como veremos a seguir, seu aspecto conteudista não mostrava preocupação com questões pedagógicas, e pela caracterização apresentada na ementa da disciplina, se adequava mais ao contexto tecnicista, seguindo os preceitos do Movimento da Matemática Moderna que não valorizava a historicidade no ensino.

Machado (2019) verificou no primeiro currículo do curso de Matemática da UEM, modificações curriculares que incluíam a disciplina de História da Matemática no segundo semestre de 1973 e caracterizavam essa disciplina como uma disciplina de conteúdo matemático, não apresentando inclinação ou abertura a discussões sobre os aspectos educacionais formativos.

No inventário arquivístico do curso de Matemática (MACHADO; TRIVIZOLI, 2019), tivemos a oportunidade de encontrar alguns documentos que nos valemos para construir uma história para essa disciplina. Dentre esses documentos, destacamos a ementa do $2^{\circ}$ semestre de 1973, um dos primeiros documentos encontrados que ilustra a presença da disciplina no curso, no qual podemos observar a lista dos pontos indicados a serem trabalhados, uma lista direta e relacionada ao aspecto conteudista dos conteúdos trabalhados. Esse ementário foi deferido em reunião do Departamento de Matemática, aos dias 22 de junho de 1973. 


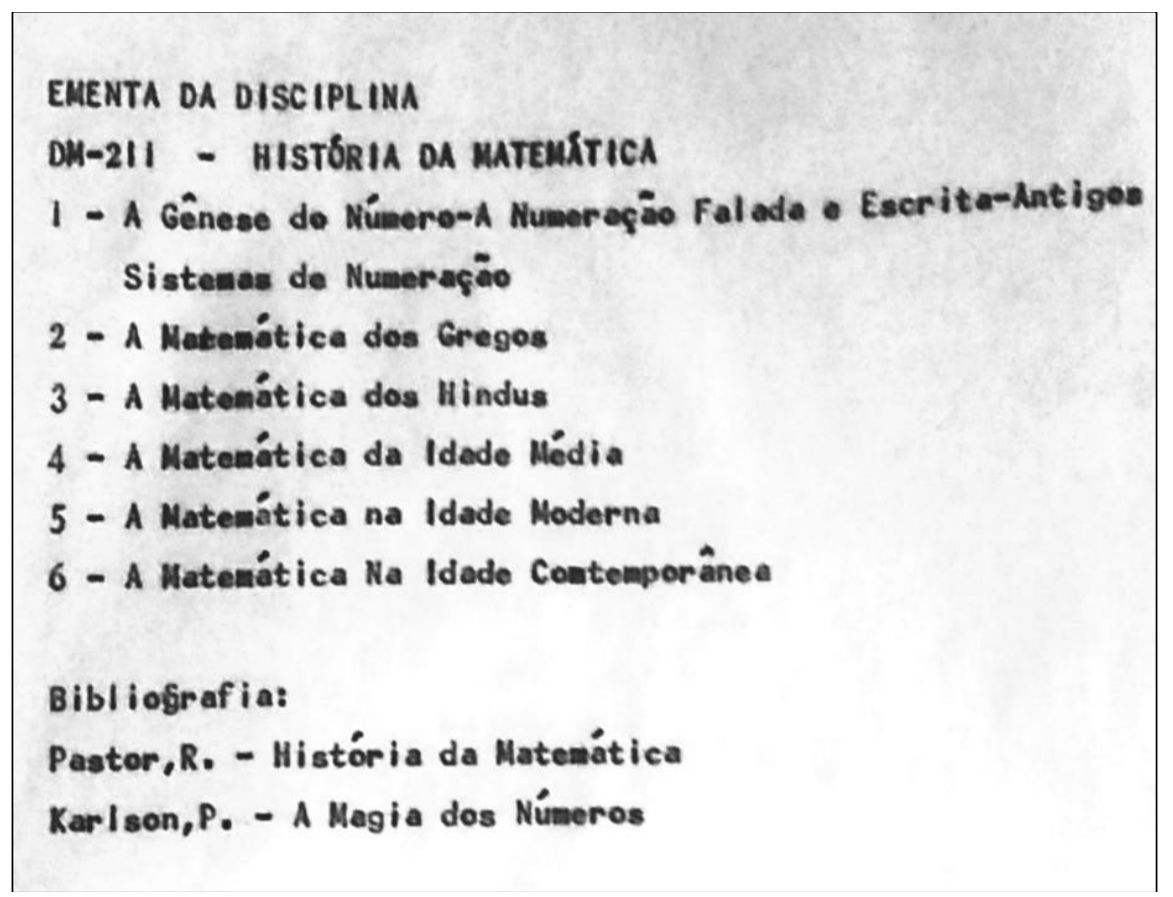

Figura 1. Ementa da disciplina História da Matemática da UEM - Ano 1973.

Fonte: Ementas Curso de Matemática UEM de 1973 (PARANÁ, 1973)

Como são poucos os documentos encontrados, destacaremos dentre os arquivos físicos do inventário informações relativas a eles, a começar pelo Histórico da disciplina História da Matemática do curso de Matemática da UEM, datado de 1974.

Para o $1^{\circ}$ Semestre de 1974 , encontramos a ementa da disciplina História da Matemática e para o $2^{\circ}$ Semestre encontramos o Plano de Curso da disciplina de História da Matemática, com carga horária de 30 horas - Noturno. Pudemos verificar dentre os documentos, que essa disciplina foi ministrada naqueles semestres pelo professor Carlos Roberto do Carmo Leite, por meio da exposição oral, estudos direcionados, palestras de outros professores, pesquisas realizadas pelos alunos, trabalhos de pesquisa apresentado por equipes com debates e verificações periódicas. Em 1974, essa disciplina contou com cerca de 28 alunos matriculados no primeiro semestre e 26 no segundo semestre.

Conforme registro, os seguintes assuntos foram ministrados no período:

"A Matemática da Pré-História - até século VI AC. As primeiras civilizações: Babilônios, Egípcios, Hindus, Chineses e Maias. Desenvolvimento da Matemática. A matemática dos Gregos. Biografia Científica dos Matemáticos da Antiguidade: Pitágoras, Euclides, Deofanto, Ptolomeu, Talles, Apolônio de Perga, Arquimedes e outros. A 
gênese do Número, 0 - Irracional, Problemas Clássicos. Helenismo. A Matemática na Idade Média: Hindus, Muçulmanos e Chineses. As primeiras grandes traduções, Desenvolvimento dos assuntos: Teoria dos conjuntos, Teoria dos números, Geometria. A Matemática do Renascimento, - época de transição ao Barroco. A Matemática na Idade Moderna. Domínio do Século XV - Desenvolvimento da Álgebra; Século XVII Renovação das Ciências Algébricas - Desenvolvimento da Geometria Analítica - A Trigonometria, os logaritmos. Surto da Análise e florescimento da Geometria. A Matemática na Idade Contemporânea Desenvolvimento da matemática. A lógica Matemática, a Física Matemática* (Resumo Geral da História da Matemática)**” (PARANÁ, HISTÓRICO DO ENSINO DA DISCIPLINA, 1974).

Em relação à primeira ementa (arquivo físico) da disciplina História da Matemática que apresentamos na figura 1 para o $2^{\circ}$ Semestre de 1973, quando analisamos os conteúdos ministrados pelos professores, verificamos a inclusão de outros conteúdos históricos, organizado pelo Departamento de Matemática para o $2^{\circ}$ Semestre de 1974. Assim, de modo a corroborar com esse discurso, trazemos o programa desta disciplina para o $1^{\circ}$ Semestre de 1974, na figura 2 da sequência para eventual consulta, que é similar a ementa oferecida para a disciplina no $2^{\circ}$ Semestre de 1974: 


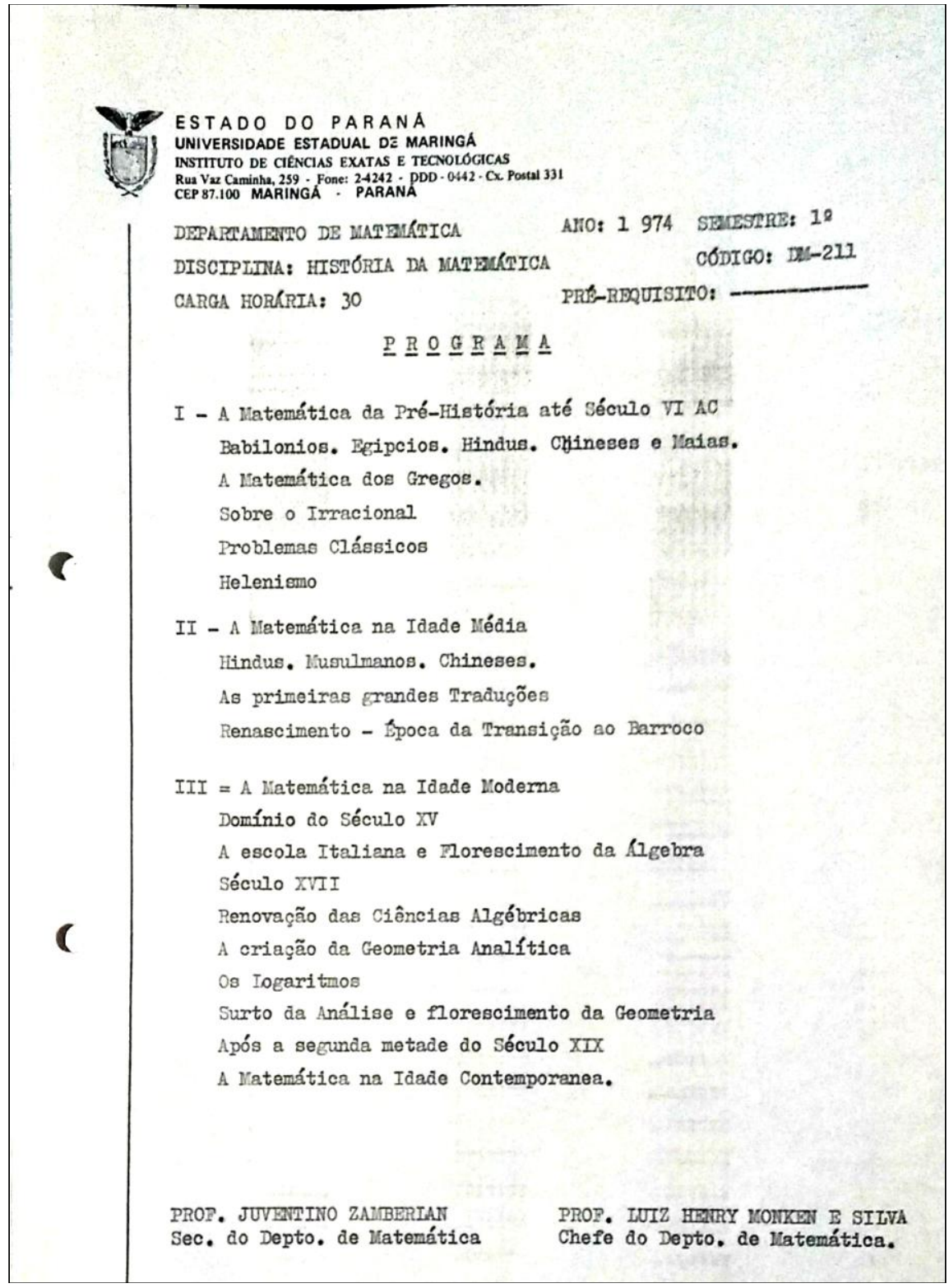

Figura 2. Programa de História da Matemática. Fonte: Paraná (1974) 
Conforme Machado (2019), a inclusão dessa disciplina para o $7^{\circ}$ Semestre do primeiro currículo do curso de Matemática da UEM e os assuntos tratados em seu núcleo, indicam conexão aos demais conteúdos que foram vistos pelos alunos no decorrer do curso, até aquele período, como possibilidade aos alunos do estudo do desenvolvimento histórico da própria Matemática.

Do período considerado para esse artigo, encontramos ainda dois documentos datados de 1979 e 1982, respectivamente. No primeiro documento, temos um Plano de Ensino que indica a trajetória da disciplina História da Matemática ministrada no $1^{\circ}$ semestre de 1979, pelo professor César Pereira, com uma carga horária semestral de 30 horas, e as estratégias de ensino que previam aulas expositivas, seminários e estudo em grupo. O objetivo da disciplina era propiciar aos alunos o conhecimento dos principais fatos da História da Matemática, assim como identificar as fases do desenvolvimento da Matemática (PARANÁ, 1979, s/f).

Por fim, destacamos o documento intitulado Relatório Final de Atividades em Disciplinas por Turma (PARANÁ, 1982), datado em 30 de junho de 1982, no qual o professor responsável pela disciplina, Elmar Waterkemper, destaca que apenas dois alunos da Matemática frequentavam essa disciplina, observando características pessoais sobre o comportamento dos alunos. Frisa que o programa era muito bom, que não existia monitoria e também não era necessário. Sobre texto, bibliografia e biblioteca para consulta da disciplina, o professor em documento destaca que muitas obras são de aquisição dos professores do Departamento de Matemática e Estatística da UEM e poucos se encontravam na biblioteca, como podemos observar o registro indicado na Figura 3:

$$
\begin{aligned}
& \text { d) Sobre reatto, bibliografia a biblíoteca }
\end{aligned}
$$

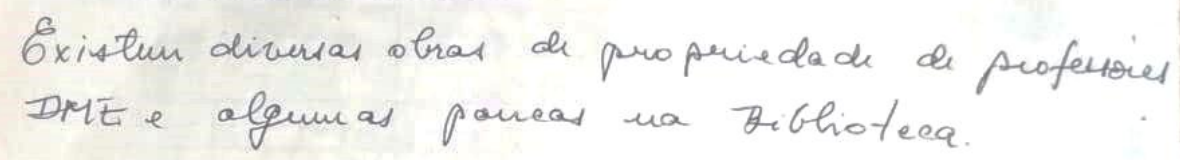

Figura 3. Relatório Final da Disciplina.

Fonte: Paraná (1982)

Contudo, lamenta ao término do documento que o novo (segundo currículo), currículo do Curso de Matemática, não apresente mais essa disciplina:

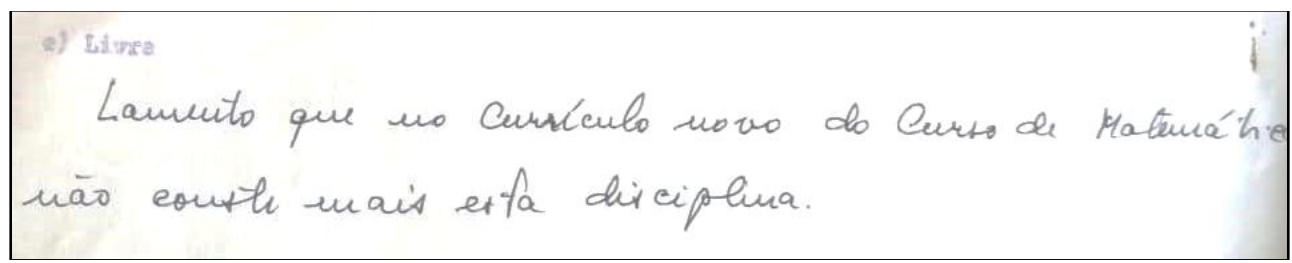

Figura 4. Relatório Final da Disciplina.

Fonte: Paraná (1982) 
Em relato ao pesquisador Araujo Neto (2016), o professor João César Guirado descreve que até aquele momento não havia disciplinas de cunho pedagógico no curso, o que reforça nossas observações que indicam que a disciplina História da Matemática apresentava uma abordagem do conteúdo sem conexões com aspectos didático-pedagógicos formativos. E destaca que:

“(...) a única disciplina que existia na época era História da Matemática, que era, aо meu ver, muito mal dada, haja vista que ela foi extinta do currículo em função dos professores que ministravam a disciplina reclamarem que não tinham condições de ministrá-la corretamente" (João César Guirado em ARAUJO NETO, 2016, p. 80).

A par desse relato, do documento de 1982 ora apresentado e da pesquisa de Machado (2019) constatamos que a disciplina não passou a figurar nos demais currículos do curso de Matemática da UEM. Entretanto, atualmente aspectos da História da Matemática estão no programa de uma das disciplinas de Teoria e Prática Pedagógica do curso de Matemática da UEM.

\section{Algumas reflexões}

A trajetória curricular da disciplina figurada nos documentos encontrados, expressa a presença curricular da disciplina no período de 1972 a 1982 na Universidade Estadual de Maringá. Contudo, entendemos que a abordagem de conteúdos matemáticos por meio da disciplina, esteja relacionada ao ambiente e as influências de ementários de outros cursos do momento, visto que os estudos direcionados a inclusão de aspectos formativos junto a disciplina História da Matemática foram fortalecidos posteriormente por meio de eventos científicos na área em meados da década de 1980.

Não podemos afirmar que a inclusão da disciplina História da Matemática no currículo do curso de Matemática da Universidade Estadual de Maringá tenha sido pensada como alteração curricular em termos de Educação Matemática, em razão do declínio da Matemática Moderna e de novas mudanças de propostas curriculares da Matemática. Essa reflexão nos remete às informações que Stamato (2013) discutiu em sua pesquisa, quanto à aplicação didática da História da Matemática no âmbito escolar, a partir da década de 1980, visto que o movimento da Matemática Moderna das décadas de 1960 e 1970 foi preconizado pelo desinteresse por abordagens históricas, devido a concepção tecnicista de ensino que prevalecia.

Sobre a caracterização que buscamos apresentar para a disciplina, as fontes históricas revelam uma inclusão curricular breve, influenciada por outros cursos de Matemática do período e uma abordagem do conteúdo sem conexões com aspectos didático-pedagógicos formativos. Mas, é importante destacar que essa é uma possível história construída a partir de vestígios históricos deixados pelo tempo, fundamentada na complexidade da pesquisa histórica, uma vez, que detalhes específicos, de zooming in, 
podem não ser encontrados e sejam necessários para alinhar o contexto. Entretanto, torna-se imprescindível assumir diferentes ângulos para compreender uma história.

\section{Bibliografia}

ARAUJO NETO, A. P. de. Um estudo histórico do curso de matemática da Universidade Estadual de Maringá: a criação e os primeiros anos. Dissertação (Mestrado em Educação em Ciência e Matemática) - Universidade Estadual de Maringá, Centro de Ciências Exatas, Programa de Pós-Graduação em Educação para a Ciência e a Matemática, Maringá - PR, 2016.

CAVALARI, M. F. Um histórico do Curso de Matemática da Faculdade de Filosofia Ciências e Letras (FFCL) da Universidade de São Paulo (USP). Revista Brasileira de História da Matemática - RBHM, Rio Claro, vol. 12, n. 16, pp. 15-30, 2012.

CERVO, A. L.; BERVIAN, P. A.; SILVA, R. Metodologia científica. 6. ed. São Paulo: Pearson, 2007.

CURY, F. G. Análise narrativa em trabalhos de História da Educação Matemática: algumas considerações. Boletim de Educação Matemática - BOLEMA, Rio Claro, vol. 23, n. 35A, pp. 59-73, 2010.

D’AMBROSIO, U. Prefácio. In: BORBA, M. C.; ARAUJO, J. de L. Pesquisa qualitativa em educação matemática. Autêntica Editora, 2004.

D’ANTONIO, S. R. Comunicação e saberes docentes: uma reflexão sobre o curso de licenciatura em matemática da Universidade Estadual de Maringá. Tese (Doutorado em Educação para a Ciência e a Matemática) - Programa de Pós-Graduação em Educação para a Ciência e a Matemática, Universidade Estadual de Maringá, Maringá, 2013.

FRAGOSO, W. C. História da matemática: uma disciplina do curso de Licenciatura em Matemática da Universidade Federal de Juiz de Fora. 2011. Dissertação (mestrado profissional em Educação Matemática) - Instituto de Ciências Exatas. Universidade Federal de Juiz de Fora, Juiz de Fora, MG, 2011.

JONES, P. S. The history of mathematics as a teaching tool: Thirty-first Yearbook. In: National Council of Teachers of Mathematics. Historical topics for the mathematics classroom. Washington, D.C, 1969.

MACHADO, S. R. A. As modificações curriculares do Curso de Matemática da Universidade Estadual de Maringá: mudanças no saber profissional do professor de matemática, 1971-1996. Dissertação (Mestrado em Educação em Ciência e Matemática) Universidade Estadual de Maringá, Centro de Ciências Exatas, Programa de Pós-Graduação em Educação para a Ciência e a Matemática, Maringá - PR, 2019.

MACHADO, S. R. A.; TRIVIZOLI, L. M. As políticas educacionais para cursos de Matemática no Brasil entre as décadas de 1960 a 1970: um olhar a partir da Revista Documenta. Revista Valore, vol. 3, pp. 62-72, 2018.

MACHADO, S. R. A.; TRIVIZOLI, L. M. Uma história para o inventário arquivístico do Departamento de Matemática da UEM. Boletim Cearense de Educação e História da Matemática, vol. 6, n. 17, pp. 56-70, 2019. 
NOBRE, S. Introdução à história da história da matemática: das origens ao século XVIII. Revista Brasileira de História da Matemática - RBHM, vol. 2, n. 3, 2002.

PARANÁ. Universidade Estadual de Maringá. Códigos de Disciplinas do Departamento de Matemática: 1971-1973. Maringá, 1973

PARANÁ. Universidade Estadual de Maringá. Histórico das disciplinas de 1974. Maringá, 1974.

PARANÁ. Universidade Estadual de Maringá. Plano de Ensino de História da Matemática. Maringá, 1979.

PARANÁ. Universidade Estadual de Maringá. Relatório Final de Atividades em Disciplinas por Turma. Maringá, 1982.

SILVA, C. M. S. A História da Matemática e os cursos de formação de professores. In: CURY, H. N. Formação de professores de matemática: uma visão multifacetada I organizado por Helena Noronha Cury. Porto Alegre: EDIPUCRS, 2001.

STAMATO, J. M. de A. A disciplina histórica da Matemática e a formação do professor de Matemática: dados e circunstâncias de sua implantação na Universidade Estadual Paulista, campi de Rio Claro, São José do Rio Preto e Presidente Prudente. Dissertação (Mestrado em Educação Matemática) - Instituto de Geociências e Ciências Exatas. Universidade Estadual Paulista, Rio Claro, 2003.

TRIVIZOLI, L. M. Intercâmbios acadêmicos matemáticos entre EUA e Brasil: uma globalização do saber. Tese (Doutorado) - Instituto de Geociências e Ciências Exatas, Universidade Estadual Paulista, Rio Claro, 2011.

TRIVIZOLI, L. M. Um panorama para a investigação em história da matemática: surgimento, institucionalização, pesquisas e métodos. Revista Paranaense de Educação Matemática, Campo Mourão, vol. 5, n. 8, pp. 189-212, 2016.

WUSSING, H. Historiography of Mathematics: Aims, Methods, Tasks. In: WOODWARD, W. R.; COHEN, R. S. (Ed.). World Views and Scientific Discipline Formation. vol. 134. Boston: Springer, pp. 63-73, 1991.

Suélen Rita Andrade Machado
Faculdade de Engenharia e Inovação Técnico
Profissional - FEITEP - Maringá - Brasil
E-mail: $\underline{\text { sumachado@gmail.com }}$
Lucieli M. Trivizoli
Universidade Estadual de Maringá - UEM -
Maringá - Brasil
E-mail: $\underline{\text { lmtrivizoli@ uem.br }}$

RBHM, Vol. 20, n 39, pp. 63-75, 2020 